\title{
Steroids and Hormones in the Environment
}

\section{Bryan O'Neil Boulanger*}

Environmental \& Water Resources Engineering Division, Zachry Department of Civil Engineering, Texas A\&M University, College Station, TX 77843, USA

Our health and wellbeing are integrally connected to our environment in ways previously unforeseen. For instance today, only 5-10 percent of all cancers are considered hereditary; resulting from an abnormality in a single gene. The other 90-95 percent of cancers are considered environmentally mediated through the way our lifestyle interacts with the food we eat, the water we drink, the air we breathe, and the soils and sediments our communities rely upon for sustenance. Because cancer mortality represents one of highest combined lifetime risks, understanding the link between the environment and cancer is critically important to the future of healthcare.

As chemical messengers, hormones play an important role in regulating our bodies. Due to their importance endocrine systems and are believed to be one of the earliest formed biological systems. Evidence supporting this belief shows up in nature today, as endocrine systems are largely conserved between mammals and non-mammalian vertebrates (such as fish). One of the best known examples of this conservative development is the similarities in function between the human estrogen receptor and estrogen receptors of organisms. Perhaps the most recognized example of this fact is the impact of feminization of male fish that live within streams receiving treated human wastewater. Therefore, exposure to estrogens from the environment (namely food and water) widely impacts biological communities.

This impact occurs because a percentage of the steroid hormones humans create to send messages within our own bodies get excreted into wastewater. In the US today about 80 percent of domestic wastewater is treated in centralized treatment systems that were never designed to remove small amounts of an organic compound (like estrogen) from large amounts of water. Steroid hormones are stable enough in the environment that a portion of the hormones pass through wastewater treatment systems and are discharged along with the treated water into a surface water body. While the amounts of steroid hormones present in the treated water are low (on the order of nanograms of steroid hormones per liter of water), it turns out that fish are very sensitive to the presence of estrogens to much lower levels than are currently observed impacted by treated wastewaters.

For many outside of the water/wastewater treatment world of emerging contaminants, my last few paragraphs may come as a surprise. However, the impact of biologically active contaminants in wastewater has been a concern within our field of study for at least the past decade. And the concern continues to grow as additional research and field studies become available. In addition to naturally occurring hormonal excretions, other endocrine disrupting chemicals also are present in treated wastewater effluents that trigger biological response in exposed organisms. These endocrine disrupting chemicals (EDCs) include some common chemicals used to formulate pesticides, plastics, flame retardants, detergents, and other personal care products. While much of the work on environmental EDCs has focused on the effect of the environmental presence of steroids and EDCs on the reproductive systems, there is mounting concern for environmental their effect on thyroid and adrenal systems, as well.

Concern over exposure to endocrine disruptors, including natural hormones, arises because the process to create potable water is a treatment loop. Treated wastewaters released to streams then are used as source waters for drinking water treatment. Effluents are also now used to recharge groundwater aquifers that may latter be used for a source of drinking water. Because many EDCs are stable, EDCs enter drinking water treatment systems where they can enter potable water. Of course exposure to EDCs in drinking water is likely a small portion of the overall exposure. The presence of EDCs in food and consumer products is likely a much higher source of exposure and should be the focus of another editorial in the future.

However, we do not fully understand whether or not exposure to steroids and hormones from the environment is a concern for people. Peter Sandman, one of the pioneers of risk communication sums up the use of toxicological studies for risk assessment nicely when he says that toxiciology is "where you collect data about what happens to small numbers of rodent exposed to high quantities of one substance at a time over a short period of time and then you try to draw conclusions about what might theoretically happen to large numbers of human being exposed to small quantities of lots of substances at one over a long period of time." In other words, we basically really do not know. However, we do know that the documented occurrence of certain types of endocrine related cancers are increasing. Why this is happening though, remains unclear.

Therefore studies seeking to understand the impact of environmental influences on endocrine function are needed. Lab and modeling studies clarifying pathways of receptor interactions with hormones and endocrine disrupting chemicals is important. Endocrine function under environmental stress is also important to consider. Success of individual patient response to clinical interventions is also important to understand, as the environment has a demonstrated response on how our bodies cope and handle endocrine related stress. Studying the impact of one endocrine systems effect the other endocrine system functions is also important to consider. I know the effect of environmental stress on endocrine system function, because I am a patient with Addison's disease (primary adrenal insufficiency) and Hashimoto's thyroiditis. And while both diseases are well understood clinically, the factors leading to their exact onset largely remain mysterious. Through both a personal and professional prospective, I believe that developing the necessary understanding to explain these factors starts with a better understanding of the role of steroids, hormones, and endocrine disruptors in the environment.

*Corresponding author: Bryan O'Neil Boulanger, Ph.D, Environmental \& Wate Resources Engineering Division, Zachry Department of Civil Engineering, Texas A\&M University, College Station, TX 77843, USA, E-Mail: bboulanger@tamu.edu

Received March 20, 2012; Accepted March 22, 2012; Published March 24, 2012

Citation: Boulanger BO (2012) Steroids and Hormones in the Environment. J Steroids Hormon Sci 3:e105. doi:10.4172/2157-7536.1000e105

Copyright: ( 2012 Boulanger BO. This is an open-access article distributed under the terms of the Creative Commons Attribution License, which permits unrestricted use, distribution, and reproduction in any medium, provided the original author and source are credited. 